\title{
Modulational Stability of Envelope Soliton in a Quantum Electron-Ion Plasma in Three Dimension - A generalised Nonlinear Schrödinger Equation in 3D
}

\author{
$j \frac{\partial \phi}{\partial t}+\sum P_{\alpha \beta} \frac{\partial^{2} \phi}{\partial x_{\alpha} \partial x_{\beta}}+Q|\phi|^{2} \phi=0$ \\ Shatadru Chaudhur* \\ Department of Physics, \\ Jadavpur University, \\ Kolkata - 700 032, \\ India \\ A. Roy Chowdhury \\ High Energy Physics Division, \\ Department of Physics, Jadavpur University, \\ Kolkata - 700 032, \\ India \\ Basudev Ghosh \\ Department of Physics, Jadavpur University, \\ Kolkata - 700 032, \\ India
}

(Dated: June 24, 2020)

\begin{abstract}
Modulational stability of envelope soliton is studied in a quantum dusty plasma in three dimension. The Krylov-Bogoliubov-Mitropolsky method is applied to the three dimension plasma governing equations. A generalised form of Nonlinear Schrödinger equation is obtained whose dispersive term has a tensorial character. Stability condition is deduced abintio and the stability zones are plotted as a function of plasma parameters.

PACS numbers: 52.27.Aj, 52.35.Mw, 52.35.Sb, 52.65.Vv, 52.35.-g

Keywords: Electron-Ion plasma, Quantum Plasma, 3D-NLS, Modulational Instability, Envelope Soliton, K-B-M method
\end{abstract}

\section{INTRODUCTION}

The study of quantum plasma was initiated mainly by the elegant works of Haas [1], Manfredi[2], Shukla 3, 4] and Brodin [5]. Up-till now many different situations have been analysed by various researchers, but all are mainly in one space and one time dimension. The basis of the formulation of quantum plasma lies in the unique phasespace quantization advocated by E.P. Wigner [6] long ago. For a long time this methodology was ignored due to the conceptual difficulty of phase space in quantum physics. But with its successful application in plasma physics, interest have been invoked and many new observations are done.

In this context one may note that many different situations of quantum plasma have been investigated in relation to the study of Nonlinear Schrödinger(NLS) equation and existence of envelope soliton but all are

\footnotetext{
* shatadru.chaudhuri12@gmail.com

$\dagger$ arc.roy@gmail.com

$\ddagger$ bsdvghosh@gmail.com
}

mainly in two dimensions. In particular the case of degenerate quantum plasma was considered by Siddki et.al[7]. Some more general situation was analysed was by B. Eliasson et.al [8, 9]. In this respect one may mention that a thermodynamically open and dissipative system was considered by Abourabia et.al[10] using the standard reductive perturbation theory, but the whole analysis was in one space dimension. The case of quantum pair-ion plasma has been studied Chaudhuri et.al[11, 12] and that of Alfven soliton in a Fermionic plasma was discussed by Keane et. al[13]. On the other-hand the situation of plasma in an external electric field was considered by Chowdhury and Pakira 14. The case of electromagnetic envelope soliton was taken up by Nusrat Jehan et. al [15]. The situation with a magnetic field was dealt by Aktar et.al[16]. But all these analysis considered a one dimensional plasma both in the classical and quantum case.

With three ideas in mind we have considered a three dimensional quantum plasma(electron-ion). A three dimensional Nonlinear Schrödinger equation is derived by taking recourse to the Krylov-BogoliubovMitropolsky method [17, 18]. The equation so derived is 
more general than the standard Nonlinear Schrödinger equation in the sense that its dispersive term has a tensorial character. To the authors knowledge this type of equation was never considered before. We have obtained the corresponding envelope soliton solution and the condition of modulational stability is obtained.

\section{FORMULATION}

Our plasma consist of positive charged ion and electron which are governed by their respective continuity and momentum equation respectively, along with the Poisson's equation. Due to small mass of electron we assume that only electrons are quantum mechanical. So, the governing equations can be written as

$$
\begin{gathered}
\frac{\partial n_{i}}{\partial t}+\vec{\nabla} \cdot\left(n_{i} \overrightarrow{u_{i}}\right)=0 \\
\frac{\partial n_{e}}{\partial t}+\vec{\nabla} \cdot\left(n_{e} \overrightarrow{u_{e}}\right)=0 \\
\frac{\partial u_{i}}{\partial t}+\left(\overrightarrow{u_{i}} \cdot \vec{\nabla}\right) \overrightarrow{u_{i}}=\frac{e}{m_{i}} \vec{E} \\
\frac{\partial u_{e}}{\partial t}+\left(\overrightarrow{u_{e}} \cdot \vec{\nabla}\right) \overrightarrow{u_{e}}= \\
-\frac{e}{m_{e}} \vec{E}-\frac{1}{m_{e} n_{e}} \vec{\nabla} P_{e}+\frac{\hbar^{2}}{2 m_{e}}\left\{\vec{\nabla}\left(\frac{\nabla^{2}\left(\sqrt{n_{e}}\right)}{\sqrt{n_{e}}}\right)\right\} \\
\vec{\nabla} \cdot \vec{E}=\frac{e}{\epsilon}\left(n_{i}-n_{e}\right)
\end{gathered}
$$

where ' $n_{i}$ ' ' $n_{e}$ ' are the densities of ions and electrons whereas ' $u_{i}$ ', ' $u_{e}$ ' are the velocities, ' $\vec{E}$ ' is the electric field. These dynamical variables are normalized in the usual manner by a scaling in the following way, $n_{i} \rightarrow$ $n_{i} / n_{i 0}, n_{e} \rightarrow n_{e} / n_{e 0}, u_{e} \rightarrow u_{e} / C_{s}, u_{i} \rightarrow u_{i} / C_{s}$ along with, $x \rightarrow x / \lambda D e, t \rightarrow \omega_{p e} t$ where, $\lambda_{D e}=\sqrt{\frac{k_{B} T_{e} \epsilon_{0}}{n_{e 0} e^{2}}}$, $\omega_{p e}=\sqrt{\frac{n_{e 0} e^{2}}{\epsilon_{0} m_{i}}}$, so that the normalized equations are written as;

$$
\begin{gathered}
\frac{\partial n_{i}}{\partial t}+\vec{\nabla} \cdot\left(n_{i} \overrightarrow{u_{i}}\right)=0 \\
\frac{\partial n_{e}}{\partial t}+\vec{\nabla} \cdot\left(n_{e} \overrightarrow{u_{e}}\right)=0 \\
\frac{\partial u_{i}}{\partial t}+\left(\overrightarrow{u_{i}} \cdot \vec{\nabla}\right) \overrightarrow{u_{i}}=\vec{E} \\
\frac{\partial u_{e}}{\partial t}+\left(\overrightarrow{u_{e}} \cdot \vec{\nabla}\right) \overrightarrow{u_{e}}=-\vec{E}-\frac{\omega_{p e}^{2}}{n_{e}} \vec{\nabla} n_{e}+H^{2}\left\{\vec{\nabla}\left(\frac{\nabla^{2}\left(\sqrt{n_{e}}\right)}{\sqrt{n_{e}}}\right)\right\}
\end{gathered}
$$

$$
\vec{\nabla} \cdot \vec{E}=\mu_{i} n_{i}-n_{e}
$$

where, $\mu_{i}=\frac{n_{i 0}}{n_{e 0}}, H^{2}=\frac{\hbar^{2} \omega_{p e}^{2}}{2 k_{B}^{2} T_{e}^{2}}$

To apply the Krylov-Bogoliubov-Mitropolsky(KBM) method [17, 18] for nonlinear wave modulation we expand all the dependent variables about their equilibrium values,

$$
\left.\begin{array}{r}
n_{e}=1+\epsilon n_{e}^{(1)}+\epsilon^{2} n_{e}^{(2)}+\epsilon^{3} n_{e}^{(3)}+\cdots \\
n_{i}=1+\epsilon n_{i}^{(1)}+\epsilon^{2} n_{i}^{(2)}+\epsilon^{3} n_{i}^{(3)}+\cdots \\
\vec{u}_{i}=\epsilon \vec{u}_{(i)}^{(1)}+\epsilon^{2} \vec{u}_{i}^{(2)}+\epsilon^{3} \vec{u}_{i}^{(3)}+\cdots \\
\vec{u}_{e}=\epsilon \vec{u}_{e}^{(1)}+\epsilon^{2} \vec{u}_{e}^{(2)}+\epsilon^{3} \vec{u}_{e}^{(3)}+\cdots \\
\vec{E}=\epsilon \vec{E}^{(1)}+\epsilon^{2} \vec{E}^{(2)}+\epsilon^{3} \vec{E}^{(3)}+\cdots
\end{array}\right\}
$$

In order to consider nonlinear excitations we assume that all the perturbed quantities in all order depend upon ' $x$ ', ' $y$ ' ' $z$ ' and ' $t$ ' through the complex amplitude $\left(\vec{a}(x, y, z, t), \vec{a}^{*}(x, y, z, t)\right)$ and the phase factor ' $\psi$ ' where, $\psi=\vec{k} \cdot \vec{r}-\omega t, \vec{r}=(x, y, z), \cdot \vec{k}=\left(k_{x}, k_{y}, k_{z}\right)$ '. As per the KBM prescription the space-time derivatives of ' $\vec{a}$ ' is written as;

$$
\left.\begin{array}{r}
\frac{\partial \vec{a}}{\partial t}=\epsilon \vec{A}_{1}+\epsilon^{2} \vec{A}_{2}+\epsilon^{3} \vec{A}_{3}+\cdots \\
\frac{\partial a_{\alpha}}{\partial x_{\beta}}=\epsilon B_{\alpha \beta}^{(1)}+\epsilon^{2} B_{\alpha \beta}^{(2)}+\epsilon^{3} B_{\alpha \beta}^{(3)} \ldots
\end{array}\right\}
$$

where $\alpha=x, y, z$ and $\beta=x, y, z$. The perturbed quantities of the electric field are written as

$$
\vec{E}=\epsilon \vec{E}^{(1)}\left(\vec{a}, \vec{a}^{*}, \psi\right)+\epsilon^{2} \vec{E}^{(2)}\left(\vec{a}, \vec{a}^{*}, \psi\right)+\epsilon^{3} \vec{E}^{(3)}\left(\vec{a}, \vec{a}^{*}, \psi\right)+\cdots
$$

From the zeroth order of $\epsilon$ we get the equilibrium condition to be $\mu_{i}=1$ i.e. $n_{i 0}=n_{e 0}$. Substituting equations 11 to 13 in equation 6 to 10 and equating like powers of $\epsilon$ we get;

$$
\begin{aligned}
& n_{e}^{(1)}=\left(\frac{\vec{k} \cdot \vec{u}_{e}}{\omega}\right) \\
& n_{i}^{(1)}=\left(\frac{\vec{k} \cdot \vec{u}_{i}}{\omega}\right)
\end{aligned}
$$

along with;

$$
\begin{aligned}
& \omega \frac{\partial u_{e x}^{(1)}}{\partial t}=-E_{x}^{(1)}-\omega_{p e}^{2} k_{x} \frac{\partial n_{e}^{(1)}}{\partial \psi}+\frac{H^{2}}{2} k_{x}^{3} \frac{\partial^{3} n_{e}^{(1)}}{\partial \psi^{3}} \\
& \omega \frac{\partial u_{e y}^{(1)}}{\partial t}=-E_{y}^{(1)}-\omega_{p e}^{2} k_{y} \frac{\partial n_{e}^{(1)}}{\partial \psi}+\frac{H^{2}}{2} k_{y}^{3} \frac{\partial^{3} n_{e}^{(1)}}{\partial \psi^{3}} \\
& \omega \frac{\partial u_{e z}^{(1)}}{\partial t}=-E_{z}^{(1)}-\omega_{p e}^{2} k_{z} \frac{\partial n_{e}^{(1)}}{\partial \psi}+\frac{H^{2}}{2} k_{z}^{3} \frac{\partial^{3} n_{e}^{(1)}}{\partial \psi^{3}}
\end{aligned}
$$




$$
\omega \frac{\partial \vec{u}_{i}^{(1)}}{\partial \psi}=\vec{E}^{(1)}
$$

Using 14 to 20, we get obtain the following equation;

$$
\left[D^{2}-\left\{\frac{\mu_{i}}{\omega^{2}}+\frac{1}{\omega^{2}}+\frac{\mu_{i} \omega_{p e}^{2}}{\omega^{4}} k^{2}\right\}+\frac{H^{2} k^{4}}{2} D^{4}\right]\left(\vec{k} \cdot \vec{E}^{(1)}\right)=0
$$

where, ' $D$ ' stands for the derivative operator ' $\frac{\partial}{\partial \psi}$ '. Substituting, $\vec{E}^{(1)}=\vec{a} \exp (i \psi)+\vec{a}^{*} \exp (-i \psi)$ we get the linear dispersion relation as follows,

$$
L \omega^{4}+M \omega^{2}+N=0
$$

where, $L=\left(1-\frac{H^{2} k^{4}}{2}\right), M=\left(1+\mu_{i}\right), N=\mu_{i} \omega_{p e}^{2} k^{2}$

The expressions for the first order quantities are;

$$
\begin{gathered}
n_{i}^{(1)}=\frac{1}{\omega^{2}}[(\vec{k} \cdot \vec{a}) \exp (i \psi)+c . c] \\
n_{e}^{(1)}=\left(\mu_{i}-\frac{1}{\omega^{2}}\right)[(\vec{k} \cdot \vec{a}) \exp (i \psi)+c . c] \\
\vec{u}_{i}^{(1)}=-\frac{1}{\omega}(\vec{a} \exp (i \psi)+c . c) \\
\vec{u}_{e}^{(1)}=\left[-\frac{1}{\omega}-\frac{\omega_{p e}^{2}}{\omega}\left(\mu_{i}-\frac{1}{\omega^{2}}\right) k^{2}-\frac{H^{2} k^{4}}{2}\left(\mu_{i}-\frac{1}{\omega^{2}}\right)\right] \\
(\vec{a} \exp (i \psi)+c . c)
\end{gathered}
$$

Similarly equating coefficients of $\epsilon^{2}$ we get;

$\omega \frac{\partial u_{e l}^{(2)}}{\partial \psi}=A_{m}^{(1)} \frac{\partial u_{e l}^{(1)}}{\partial a_{m}}+\omega_{p e}^{2}\left(B_{l m}^{(1)} \frac{\partial n_{e}^{(1)}}{\partial a_{m}}\right)-\frac{H^{2}}{2} k_{l}^{3} \frac{\partial^{3} n_{e}^{(2)}}{\partial \psi^{3}}+$

$\frac{H^{2}}{2} n_{e}^{(1)} k_{l}^{3} \frac{\partial^{3} n_{e}^{(1)}}{\partial \psi^{3}}+\omega_{p e}^{2} k_{l} \frac{\partial n_{e}^{(2)}}{\partial \psi}-\omega_{p e}^{2} k_{l} n_{e}^{(1)} \frac{\partial n_{e}^{(1)}}{\partial \psi}+$

$k_{l} u_{e l}^{(1)} \frac{\partial u_{e l}^{(1)}}{\partial \psi}+E_{l}^{(2)}$

with sum over repeated index and $(l=x, y, z ; m=$ $x, y, z)$. Similarly;

$$
\omega \frac{\partial u_{i l}^{(2)}}{\partial \psi}=A_{m} \frac{\partial u_{i l}^{(1)}}{\partial a_{l}}+k_{l} u_{i l}^{(l)} \frac{\partial u_{i l}^{(1)}}{\partial \psi}-E_{l}^{(2)}
$$

$$
\begin{array}{r}
\omega \frac{\partial n_{e}^{(2)}}{\partial \psi}=A_{l}^{(1)} \frac{\partial n_{e}^{(1)}}{\partial a_{l}}+B_{x l}^{(1)} \frac{\partial u_{e x}^{(1)}}{\partial a_{l}}+k_{l} \frac{\partial u_{e l}^{(2)}}{\partial \psi}+B_{y l} \frac{\partial u_{e y}^{(1)}}{\partial a_{l}} \\
+k_{l} \frac{\partial}{\partial \psi}\left(n_{e}^{1} u_{e l}^{1}\right)+B_{z l} \frac{\partial u_{e z}}{\partial a_{l}}
\end{array}
$$

$$
\begin{array}{r}
\omega \frac{\partial n_{i}^{(2)}}{\partial \psi}=A_{l}^{(1)} \frac{\partial n_{i}^{(1)}}{\partial a_{l}}+B_{x l}^{(1)} \frac{\partial u_{i x}^{(1)}}{\partial a_{l}} \\
+k_{l} \frac{\partial u_{i l}^{(2)}}{\partial \psi}+B_{y l} \frac{\partial u_{i y}^{(1)}}{\partial a_{l}} \\
+k_{l} \frac{\partial}{\partial \psi}\left(n_{i}^{1} u_{i l}^{1}\right)+B_{z l} \frac{\partial u_{i z}^{(1)}}{\partial a_{l}}
\end{array}
$$

Lastly from the Poisson's equation we get,

$$
\begin{array}{r}
k_{l} \frac{\partial E_{l}^{(2)}}{\partial \psi}+B_{l x}^{(1)} \frac{\partial E_{x}^{(1)}}{\partial a_{l}}+B_{l y}^{(1)} \frac{\partial E_{y}^{(1)}}{\partial a_{l}}+B_{l z}^{(1)} \frac{\partial E_{z}^{(1)}}{\partial a_{l}}= \\
\mu_{i} n_{i}^{(2)}-n_{e}^{(2)}
\end{array}
$$

Using the equations (23) to (30) we get;

$$
\begin{aligned}
\left(D^{3}+D\right)\left(\vec{k} \cdot \vec{E}^{(2)}\right)= & \frac{\chi}{\alpha^{\prime}}\left[A_{1}+\overrightarrow{v_{g}} \cdot \stackrel{\leftrightarrow}{B}_{1}\right] \exp (i \psi)+ \\
& \frac{1}{\alpha^{\prime}} \text { Terms proportional to } \exp (2 i \psi)
\end{aligned}
$$

The above equation in order to be secularity free, requires $\left[\vec{A}_{1}+\vec{v}_{g} \cdot \overleftrightarrow{B}_{1}\right]=0$. Here, ' $\vec{v}_{g}$ ' is the group velocity and is given by

$$
\vec{v}_{g}=\frac{\alpha^{\prime}}{\chi}
$$

where, $\alpha^{\prime}=\left(2 \omega_{p e}^{2} \omega^{2}+2 \omega_{p e}^{2} \mu_{i}+2 H^{2} k^{2} \omega^{2}+2 H^{2} k^{2} \mu_{i}\right) \vec{k}$ $\chi=2 \omega^{2}+\mu_{i}-2 \omega \omega_{p e} k^{2}-H^{2} k^{4} \omega-1$

Next proceeding to the perturbation terms which are third order in $\epsilon$, we get;

$$
-\sigma\left\{1+\frac{\partial^{2}}{\partial \psi^{2}}\right\}\left(\vec{k} \cdot \vec{E}^{(3)}\right)=L(1,2)
$$

where,

$$
\begin{array}{r}
L(1,2)=\frac{\mu_{i}}{\omega^{2}}\left(\omega^{2}-\omega_{p e}^{2} k^{2}\right) J_{1}(1,2)- \\
\left(\omega^{2}-\omega_{p e}^{2} k^{2}\right) F(1,2)-\frac{H^{2} k^{4}}{2} I_{1}(1,2)+ \\
\frac{\mu_{i}}{\omega^{2}} \frac{\partial^{2} J_{1}(1,2)}{\partial \psi^{2}}+G(1,2)
\end{array}
$$

with $\sigma=\left\{1+\frac{\mu_{i}}{\omega^{2}}\left(\omega^{2}-\omega_{p e}^{2} k^{2}\right)\right\}$ In the same way as before, in order to have equation(34) to be secularity free, we set

$\chi\left(A_{2}+\vec{v}_{g} \cdot \overleftrightarrow{B}_{2}\right)+i \Lambda_{\alpha \beta}\left(B_{\alpha \beta} \frac{\partial B_{\alpha \beta}}{\partial a_{l}}\right)+i Q_{0}|\vec{k} \cdot \vec{a}|^{2}+i R_{0}(\vec{k} \cdot \vec{a})=0$

Thus by suitable scale transformation and taking $\vec{k} \cdot \vec{a}=$ $\phi$ we get the standard Nonlinear Schrödinger equation given as;

$$
j \frac{\partial \phi}{\partial \tau}+P_{\alpha \beta} \frac{\partial^{2} \phi}{\partial \xi_{\alpha} \partial \xi_{\beta}}+Q|\phi|^{2} \phi+R \phi=0
$$




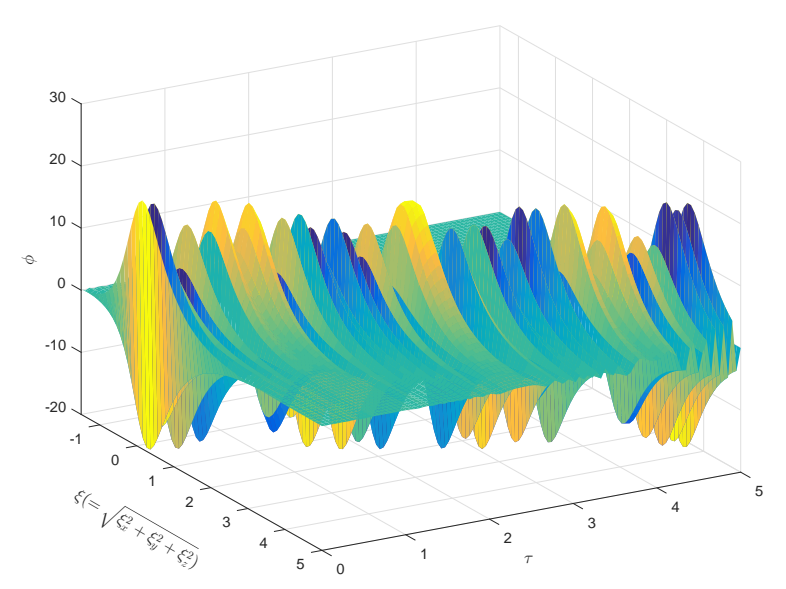

FIG. 1: The Envelope Soliton or the Envelope Solitary wave

where it should be noted that $P_{\alpha \beta}=-\frac{\Lambda_{\alpha \beta}}{\chi}, Q=-Q_{0} / \chi$ and $R=-R_{0} / \chi$. The expressions for $\Lambda_{\alpha \beta}, Q_{0}, R_{0}$ are given in the appendix.

Equation (36) is the generalised form of Nonlinear Schrödinger equation in three dimension. Expression for $F(1,2), J(1,2)$ and $I(1,2)$ are all given in the appendix. On should note that in this equation the usual Laplacian term is replaced a second order operator where the coefficients of each second order space derivative are different. In this equation the last term ' $R a$ ', known as the linear interaction term, causes simply a phase shift. Using a simple substitution $\vec{a} \rightarrow \vec{a} \exp [i R \tau]$ we get the standard Nonlinear Schrödinger equation in 3D as

$$
j \frac{\partial \phi}{\partial \tau}+P_{\alpha \beta} \frac{\partial^{2} \phi}{\partial \xi_{\alpha} \partial \xi_{\beta}}+Q|\phi|^{2} \phi=0
$$

It should be noted that in the above equations (for example equations (36) or (37)) and also in other places in this article we have used $j=\sqrt{-1}$ instead of ' $i$ ' to avoid confusion with notation of ions.

We know that, when there is a nonlinear evolution of a wave, it may so happen that te nonlinear effect is balanced by the dispersion effect and a stable wave structure is formed. Actually the application of the external electric field on the plasma causes a local depression in density called a 'caviton'. Plasma waves trapped in this cavity then form an isolated structure called an 'envelope soliton' which is one of the solution of the equation( 37). A graphical representation of such a soliton is depicted by figure( (1) and its corresponding contour plot is given in figure( 2).

\section{DISPERSION AND STABILITY}

To proceed further we start with the dispersion relation (21). Here we have plotted ' $\omega$ ' as a function of the

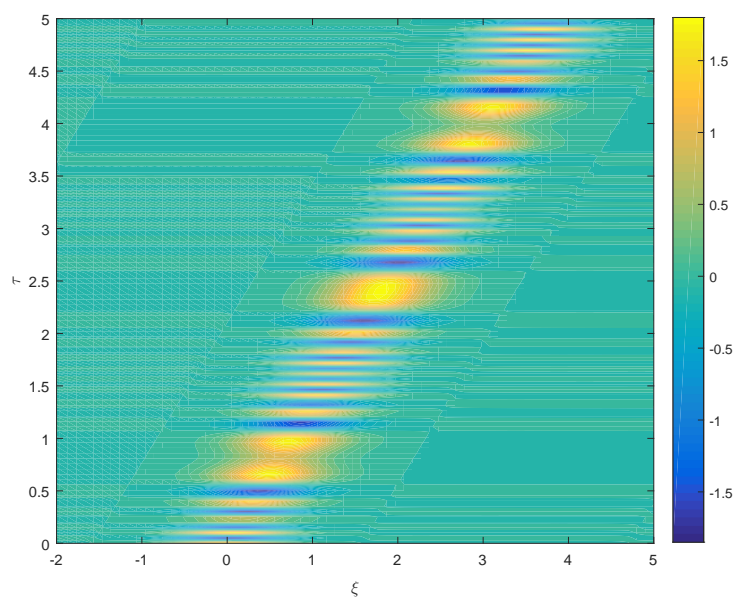

FIG. 2: The contour plot of the envelope soliton
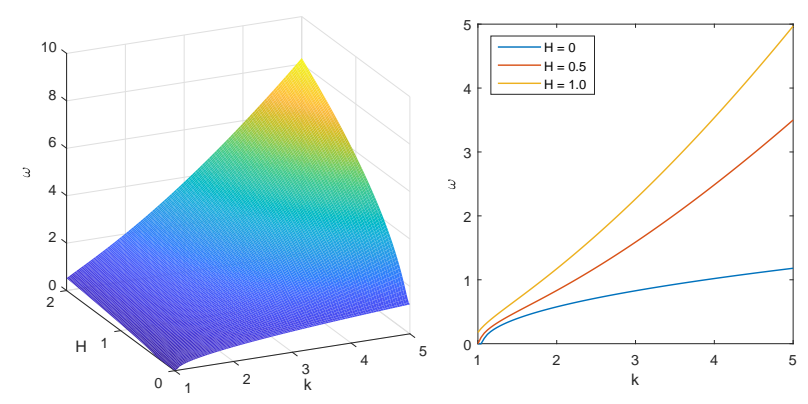

FIG. 3: The plot of dispersion relation, here

$$
k=\sqrt{k_{x}^{2}+k_{y}^{2}+k_{z}^{2}}
$$

wave vector $k\left(=\sqrt{k_{x}^{2}+k_{y}^{2}+k_{z}^{2}}\right)$ in figure( (3). One may observe that the trend is quite similar to that observed in one dimension. Here we have shown three different curves pertaining to three separate values of ' $H$ '. The next important quantity is the group velocity ' $\vec{v}_{g}$ ' as given in equation (32). Here also in the figure $4(a, b, c)$ we have depicted the variation of $v_{g x}$ with respect to $k_{x}, k_{y}$ and $k_{z}$. One may note that where as $v_{g x}$ has a growing behaviour with respect to $k_{x}$, it has a decreasing nature when seen with respect to $k_{y}$ and $k_{z}$. This event has also been repeated for $v_{g y}$ and $v_{g z}$. Our next important feature is the tensorial components of the dispersive terms. Let us start with $P_{x x}$ and consider its variation with $k_{x}$ ,$k_{y}$ and $k_{z}$ by subplot( (5). The negative and positive regions are explicitly exhibited in the contour plot. A similar behaviour is seen for $P_{y y}$ in subplot( [6). Since $P_{\alpha \beta}$ is a symmetric tensor, hence we obtained a of $P_{z z}$ similar to that of $P_{x x}$ and $P_{y y}$. On the other-hand due to the symmetric behaviour of $P_{\alpha \beta}$ the variation of the off-diagonal elements of $P_{\alpha \beta}$ are similar (i.e. $P_{\alpha \beta}=P_{\beta \alpha}$ ). 

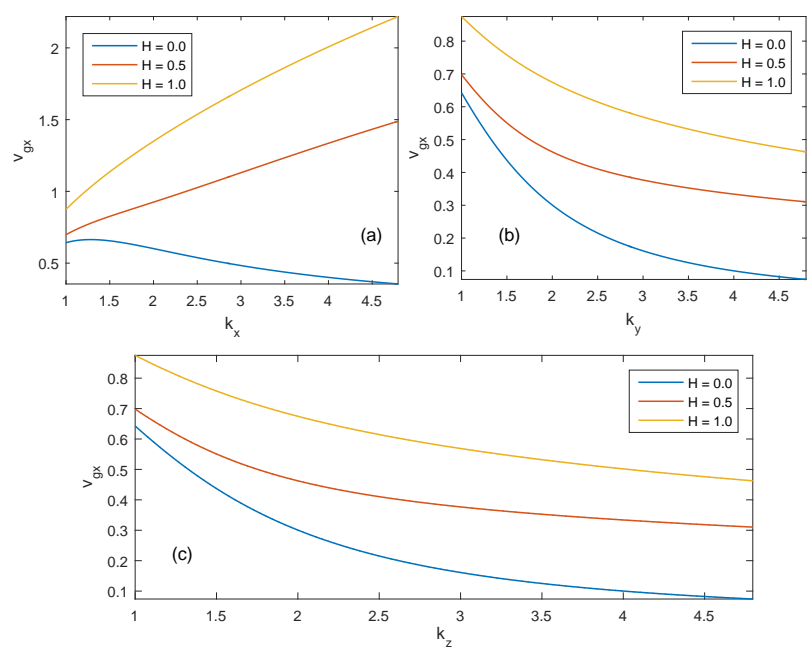

FIG. 4: The plot of Group Velocity

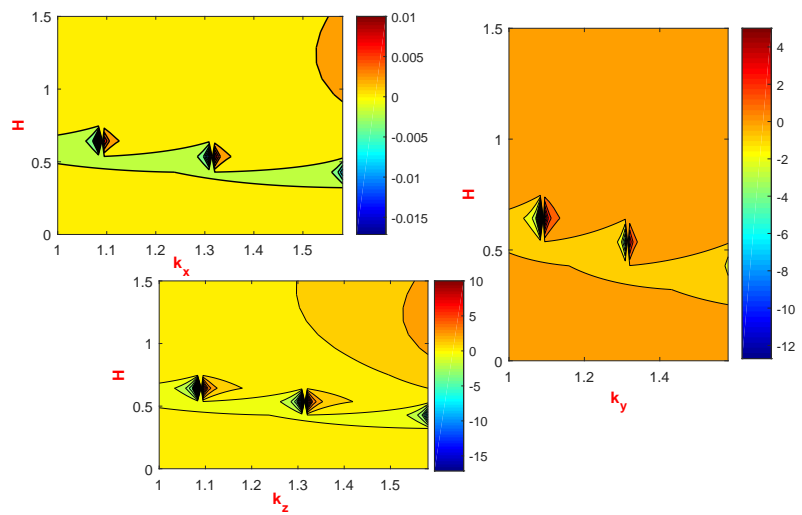

FIG. 5: Contour plot of the dispersive coefficient component $P_{x x}$. The variation is shown with respect to the quantum diffraction parameter $H$ and wave $\operatorname{vectors}\left(k_{x}, K_{y}\right.$ and $\left.k_{z}\right)$.

Thus in this communication we have shown the variation of $P_{x y}$ and $P_{x z}$ only, depicted in the figures( (7) and( 8) respectively. The variation of the nonlinear coefficient $Q$ is depicted by the figure( (9) with respect to $k_{x}$. Since $Q$ is a scalar, so its variation with $k_{y}$ and $k_{z}$ were similar as the variation with respect to $k_{x}$.

To explore further we try to investigate the modulational instability of the system (37).

For that we set

$$
\phi=\left(\Phi_{0}+\delta \Phi(\zeta)\right) \exp [-i \Delta \tau]
$$

$\Phi_{0}$ being the carrier wave amplitude, $K$ and $\Omega$ the modulational wave number and frequency respectively and $\zeta=\vec{K} \cdot \vec{\xi}-\Omega \tau$

Using this expression of ' $\Phi$ ' in equation (38), from the zeroth order term of $\delta \Phi$ we get;

$\Delta=-Q\left|\Phi_{0}\right|^{2}$
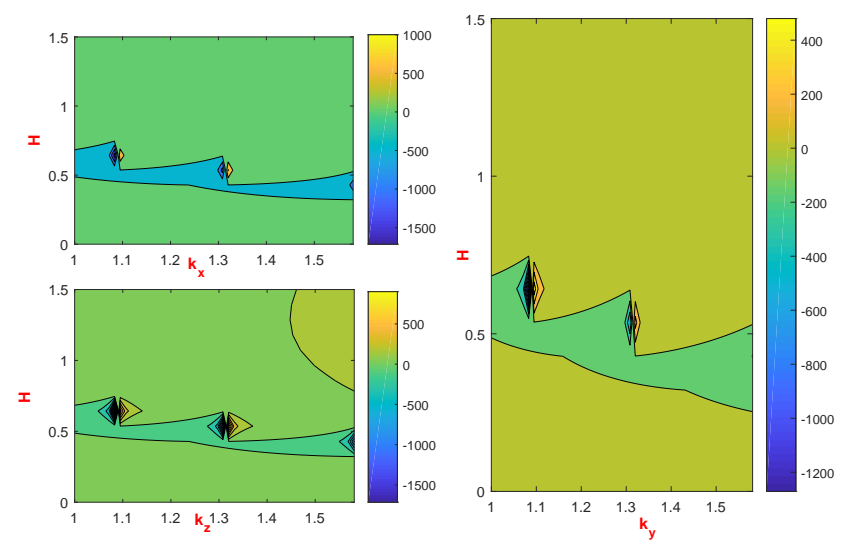

FIG. 6: Contour plot of the dispersive coefficient component $P_{y y}$. The variation is shown with respect to the quantum diffraction parameter $H$ and wave $\operatorname{vectors}\left(k_{x}, K_{y}\right.$ and $\left.k_{z}\right)$.

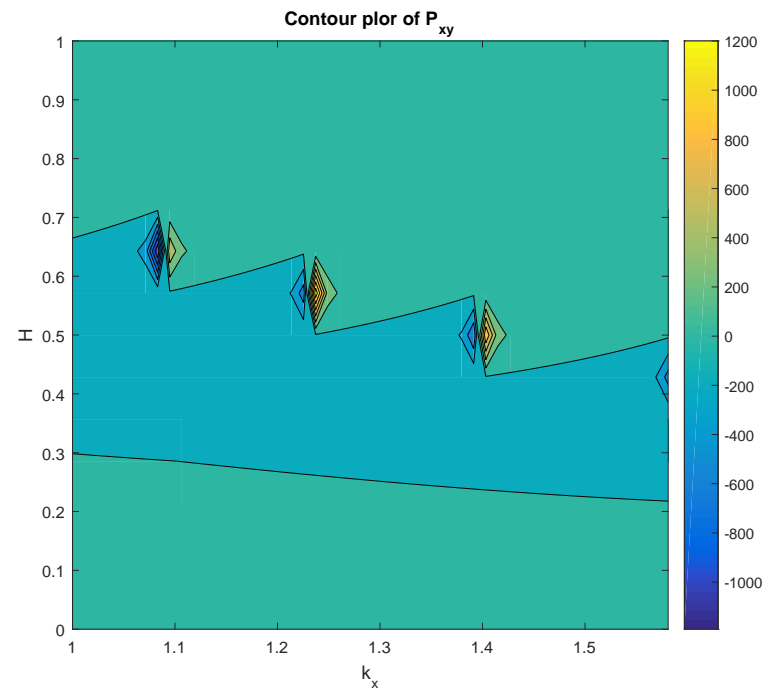

FIG. 7: Contour plot of the dispersive coefficient component $P_{x y}$. The variation is shown with respect to the quantum diffraction parameter $H$ and wave $\operatorname{vector}\left(k_{x}\right)$.

And from the first order term of $\delta \Phi$ we get;

$$
\begin{aligned}
& j \frac{\partial}{\partial \tau}(\delta \Phi)+\Delta(\delta a)+P \frac{\partial^{2}}{\partial \xi_{\alpha} \partial \xi_{\beta}}(\delta \Phi)+Q\left|a_{0}\right|^{2}\left(\delta \Phi+\delta \Phi^{*}\right) \\
& +Q\left|\Phi_{0}\right|^{2}(\delta \Phi)=0
\end{aligned}
$$

where, $\delta \Phi^{*}$ is the complex conjugate of $\delta \Phi$. Setting, $\delta \Phi=$ $\mathcal{U}+i \mathcal{V}$ and using the expression of ' $\Delta$ ' obtained from the zeroth order term of $\delta \Phi$, one gets by separating the real 


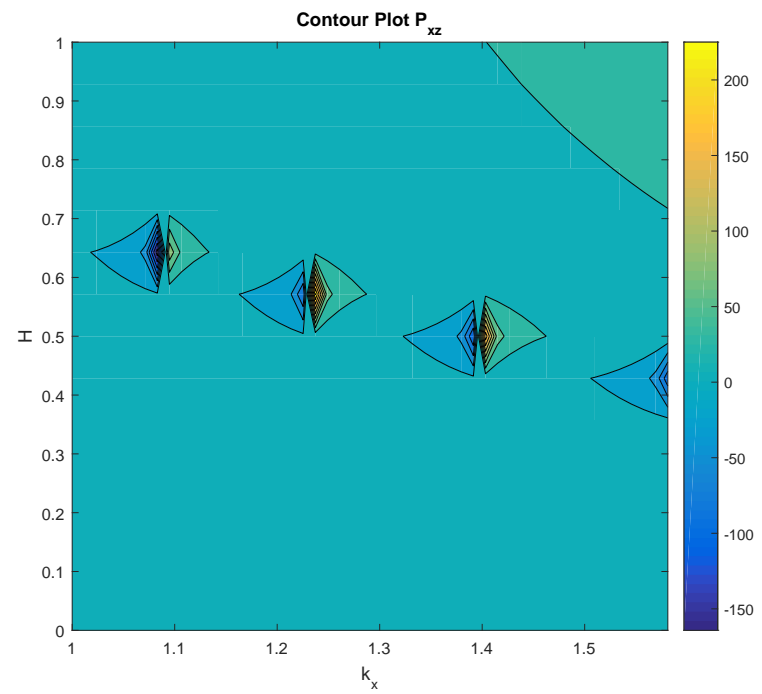

FIG. 8: Contour plot of the dispersive coefficient component $P_{x z}$. The variation is shown with respect to the quantum diffraction parameter $H$ and wave $\operatorname{vector}\left(k_{x}\right)$.

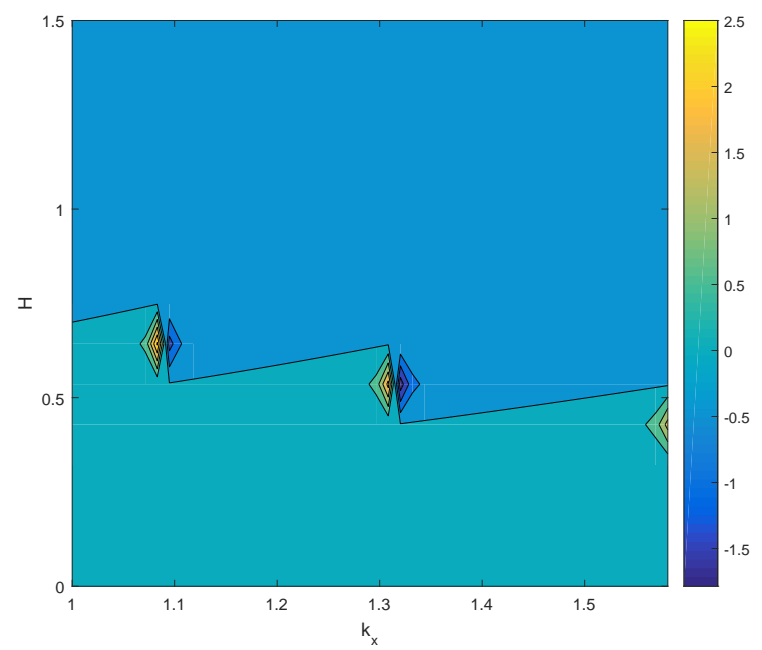

FIG. 9: Contour plot of the nonlinear coefficient $Q$. The variation is shown with respect to the quantum diffraction parameter $H$ and wave vector $\left(k_{x}\right)$.

and imaginary parts;

$$
\left.\begin{array}{r}
\frac{\partial \mathcal{U}}{\partial \tau}+P_{\alpha \beta} \frac{\partial^{2} \mathcal{V}}{\partial \xi_{\alpha} \xi_{\beta}}=0 \\
-\frac{\partial \mathcal{V}}{\partial \tau}+P_{\alpha \beta} \frac{\partial^{2} \mathcal{U}}{\partial \xi_{\alpha} \xi_{\beta}}+2 Q \mathcal{U}\left|\Phi_{0}\right|^{2}=0
\end{array}\right\}
$$

Next assuming plane wave solutions of $\mathcal{U}$ and $\mathcal{V}$ i.e.,

$$
\left.\begin{array}{l}
\mathcal{U}=\mathcal{S} \exp (j[\vec{K} \cdot \vec{\xi}-\Omega \tau]) \\
\mathcal{V}=\mathcal{J} \exp (j[\vec{K} \cdot \vec{\xi}-\Omega \tau])
\end{array}\right\}
$$

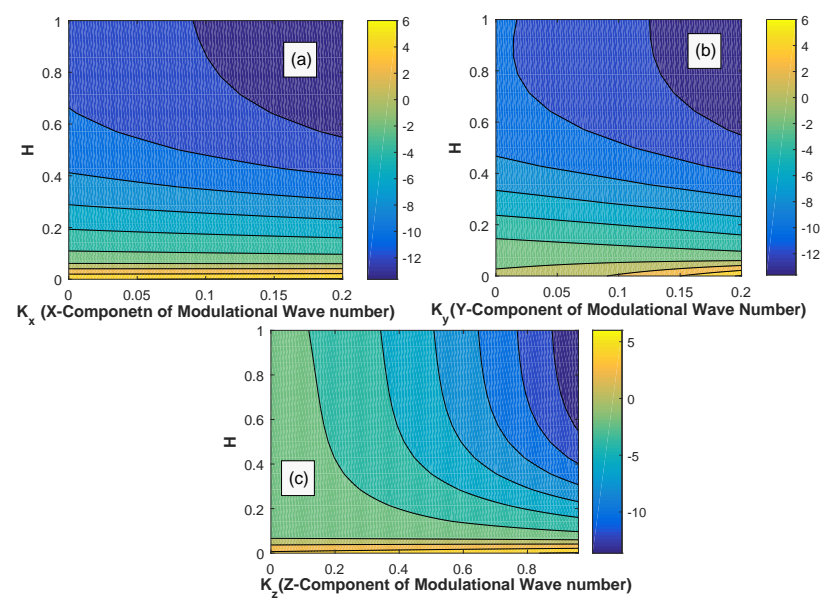

FIG. 10: Contour plot of the $\alpha / Q$. The variation is shown with respect to the quantum diffraction parameter $H$ and modulational wave $\operatorname{number}\left(K_{x}, K_{y}\right.$ and $K_{z}$ respectively).

These linearised equations leads to

$$
\left|\begin{array}{cc}
j \Omega & K_{\alpha} K_{\beta} P_{\alpha \beta} \\
-K_{\alpha} K_{\beta} P_{\alpha \beta}+2 Q\left|\Phi_{0}\right|^{2} & j \Omega
\end{array}\right|=0
$$

which reduces to,

$$
\Omega^{2}+2 \alpha Q\left|\Phi_{0}\right|^{2}-\alpha^{2}=0
$$

where, $\alpha=K_{x} K_{x} P_{x x}+K_{x} K_{y} P_{x y}+K_{x} K_{z} p_{x z}+$ $K_{y} K_{x} P_{y x}+K_{y} K_{y} P_{y y}+K_{y} K_{z} P_{y z}+K_{z} K_{x} P_{z x}+$ $K_{z} K_{y} P_{z y}+K_{z} K_{z} P_{z z}$

solution of (41) yields; $\Omega=\sqrt{\alpha^{2}-\alpha\left(2 Q\left|\Phi_{0}\right|^{2}\right)}$. So that condition of stability turns out to be :: if $\left(\frac{\alpha}{2 Q\left|\Phi_{0}\right|^{2}}<1\right)$ then the wave is unstable and if $\left(\frac{\alpha}{2 Q\left|\Phi_{0}\right|^{2}}>1\right)$ then the wave is stable. To ascertain this condition numerically we have plotted the contour plot of $\left(\frac{\alpha}{Q}\right)$ for various values of $H$ and $K_{x}, K_{y}$ and $K_{z}$ in subplot([10, b,c).

We see that in the subplot( $10 \mathrm{k}, \mathrm{c})$, for the $H \gtrsim 0.1$ and for all values of modulational wave number $K_{l}$ the ratio ' $\alpha / Q$ ' becomes less than 1 and the wave instability sets in. While in figure (10b) though the behaviour of $\alpha / Q$ is bit different from that of $(\mathrm{a}, \mathrm{c})$ but here also the instability set in for $H \geqslant 0.2$. Thus we see that the quantum diffraction parameter has a good impact on the stability of the wave.

At this point we may note that the modulational stability condition is slightly changed in this $3 \mathrm{D}$ case.

\section{DISCUSSIONS AND CONCLUSIONS}

In our above analysis we have studied the evolution of envelope soliton using the Krylov-BogoliubovMitropolsky method. In this case we have considered 
a quantum hydrodynamic(QHD) model. The Nonlinear Schrödinger equation deduced in this case is 3dimensional where the group velocity is $3 \mathrm{D}$-vector quantity and the dispersive coefficient ' $P_{\alpha \beta}$ ' is a symmetric tensor. Both the dispersion relation and the group velocity of the system has a clear variation not only with the wave vector $\left(k_{l}\right)$ but also with the quantum diffraction parameter $(H)$. Next we analyzed the modulational stability of the system. The phenomenon of modulational instability is one of the basic topic to be studied in the domain of non-linear plasma physics. Initially it was an idea put forwarded by Benjawin and Feir 19] in case of water waves and by Bespalov and Talanov 20] for electromagnetic wave application in many branches of physics such as, nonlinear optics, condensed matter physics, etc. When the monochromatic wave(or quasi counter part) as it propagates through the nonlinear dispersive media gives rise to two side-bands. The whole phenomenon is actually related to the role this side-bands play in the growth and decay of the propagating side-bands. With the help of nonlinear dispersion relation( 41) we have found the stability criteria. We see that when the ratio ' $(\alpha / Q)>1$ ' the carrier wave is modulationally 'stable' while for ' $(\alpha / Q)<1$ ' the carrier wave becomes modulationally 'unstable'. The values of the plasma parameters used for the numerical analysis are as follows: $n_{i 0}=n_{e 0} \sim(1-4) \times 10^{14}(\text { metre })^{-1}$ and $\omega_{p e} \sim 10^{7} \mathrm{~Hz}$.

\section{Appendix A}

$$
\begin{aligned}
& J_{1}(1,2)=\omega A_{l}^{(1)} \frac{\partial n_{i}^{(2)}}{\partial a_{l}}+\omega A_{l}^{(2)} \frac{\partial n_{i}^{(1)}}{\partial a_{l}}+\omega B_{l m}^{(1)} \frac{\partial u_{i l}^{(2)}}{\partial a_{m}}+ \\
& k_{l} A_{l}^{(1)} \frac{\partial u_{i l}^{(2)}}{\partial a_{l}}+k_{l} u_{i l}^{(1)} B_{l m}^{(1)} \frac{\partial u_{i l}^{(1)}}{\partial a_{l}}+k^{2} u_{i l}^{(2)} \frac{\partial u_{i l}^{(1)}}{\partial \psi}+ \\
& k_{l} u_{i l}^{(1)} B_{l m}^{(1)} \frac{\partial u_{i l}^{(1)}}{\partial a_{m}}+k_{l} k_{m} u_{i l}^{(1)} \frac{\partial u_{i m}^{(2)}}{\partial \psi}+k_{l} k_{m} u_{i m}^{(2)} \frac{\partial u_{i l}^{(1)}}{\partial \psi}+ \\
& \omega B_{l m}^{(1)} \frac{\partial}{\partial a_{m}}\left(n_{i}^{(1)} u_{i l}^{(1)}\right)
\end{aligned}
$$

$$
I_{1}(1,2)=-k_{l} \frac{\partial^{4} E_{l}^{(3)}}{\partial \psi^{4}}-B_{l m}^{(1)} \frac{\partial^{4} E_{l}^{(2)}}{\partial a_{m} \partial \psi^{3}}-B_{l m}^{(2)} \frac{\partial^{4} E_{l}^{(1)}}{\partial a_{m} \partial \psi^{3}}
$$

$$
\begin{gathered}
F(1,2)=-k_{l} \frac{\partial^{2} E_{l}^{(3)}}{\partial \psi^{2}}+B_{l m}^{(1)} \frac{\partial^{2} E_{l}^{(2)}}{\partial a_{l} \partial \psi}+B_{l m}^{(2)} \frac{\partial^{2} E_{l}^{(1)}}{\partial a_{l} \partial \psi} \\
J(1,2)=J_{1}(1,2)-k_{l} E_{l}^{(3)}
\end{gathered}
$$

where the indices ' $l$ ', ' $m$ ' denotes $(x, y, z)$ as used in the other sections of the article.

$$
\begin{aligned}
& \Lambda_{x x}=12\left(\omega^{2}\right)\left(v_{g y}^{2}\right)-6\left(H^{2}\right)\left(\omega^{2}\right)\left(v_{g y}^{2}\right)\left(k^{4}\right)- \\
& 6\left(H^{2}\right)\left(\omega^{4}\right)\left(k_{y}^{2}\right)-16\left(H^{2}\right)\left(\omega^{3}\right) v_{g y}\left(k^{2}\right) k_{y}+2\left(v_{g y}^{2}\right) \\
& +2 \mu_{i}\left(v_{g y}^{2}\right)+2 \mu_{i}\left(\omega_{p e}^{2}\right) \\
& \Lambda_{y y}=12\left(\omega^{2}\right)\left(v_{g y}^{2}\right)-6\left(H^{2}\right)\left(\omega^{2}\right)\left(v_{g y}^{2}\right)\left(k^{4}\right)- \\
& 6\left(H^{2}\right)\left(\omega^{4}\right)\left(k_{y}^{2}\right)-16\left(H^{2}\right)\left(\omega^{3}\right) v_{g y}\left(k^{2}\right) k_{y}+2\left(v_{g y}^{2}\right) \\
& +2 \mu_{i}\left(v_{g y}^{2}\right)+2 \mu_{i}\left(\omega_{p e}^{2}\right) \\
& \Lambda_{z z}=12\left(\omega^{2}\right)\left(v_{g z}^{2}\right)-6\left(H^{2}\right)\left(\omega^{2}\right)\left(v_{g z}^{2}\right)\left(k^{4}\right)- \\
& 6\left(H^{2}\right)\left(\omega^{4}\right)\left(k_{z}^{2}\right)-16\left(H^{2}\right)\left(\omega^{3}\right) v_{g z}\left(k^{2}\right) k_{z}+2\left(v_{g z}^{2}\right)+ \\
& 2 \mu_{i}\left(v_{g z}^{2}\right)+2 \mu_{i}\left(\omega_{p e}^{2}\right) \\
& \Lambda_{x y}=12\left(\omega^{2}\right) v_{g x} v_{g y}-6\left(H^{2}\right)\left(\omega^{2}\right) v_{g y} v_{g x}\left(k^{4}\right)- \\
& 8\left(H^{2}\right)\left(\omega^{3}\right) v-g x k_{y} k^{2}-8\left(H^{2}\right) v-g y k_{x} k^{2}- \\
& \left(\left(H^{2}\right) / 2\right)\left(\omega^{4}\right)\left(4\left(k_{x} k_{y}+k_{x} k_{z}\right)\right)+2 v_{g y} v_{g x}+ \\
& 2 \mu_{i} \operatorname{vgyv}_{g x}=\Lambda_{y x} \\
& \Lambda_{x z}=12\left(\omega^{2}\right) v_{g x} v_{g z}-\left(\left(H^{2}\right) / 2\right) 12\left(\omega^{2}\right) v_{g x} v_{g z} k^{4}- \\
& \left(\left(H^{2}\right) / 2\right) 4\left(\omega^{3}\right) v_{g z} 4 k_{x} k^{2}-4\left(\left(H^{2}\right) / 2\right) 4\left(\omega^{3}\right) v_{g x} k_{z} k^{2}- \\
& \left(\left(H^{2}\right) / 2\right)\left(\omega^{4}\right)\left(4\left(k_{x} k_{z}+k_{z} k_{y}\right)\right)+12 v_{g x} v_{g z}+12 \mu_{i} v_{g x} v_{g z}=\Lambda_{z x} \\
& Q_{0}=-\left(\left(\omega^{5}\right) /\left(12\left(k^{6}\right)\right)\right)\left(3 \omega_{p e}^{2}+2\left(\omega^{2}\right) k^{2}+\left(\left(H^{2}\right)\left(k^{10}\right)\right)-\right. \\
& \left.2 \mu_{i}\left(H^{2}\right)\left(k^{6}\right)+6\left(k^{8}\right)+76 k^{2}+5\right)
\end{aligned}
$$

\section{ACKNOWLEDGEMENT}

One of the author Shatadru Chaudhuri (SC) is grateful to the Department of Physics, Jadavpur University for a fellowship under the scheme RUSA. SC would also like to thank Mr. Jyotirmoy Goswami and Mr. Jit Sarkar for thier support and inspiration. 
[1] Fernando Haas (auth.). Quantum Plasmas: An Hydrodynamic Approach. Springer Series on Atomic, Optical, and Plasma Physics 65. Springer-Verlag New York, 1 edition, 2011.

[2] Giovanni Manfredi. How to model quantum plasma. Fields Institute Communications, 46, 062005.

[3] Padma K Shukla and B Eliasson. Nonlinear aspects of quantum plasma physics. Physics-Uspekhi, 53(1):51-76, jan 2010.

[4] P K Shukla and B Eliasson. Recent developments in quantum plasma physics. Plasma Physics and Controlled Fusion, 52(12):124040, nov 2010.

[5] G. Brodin, M. Marklund, and G. Manfredi. Quantum plasma effects in the classical regime. Phys. Rev. Lett., 100:175001, Apr 2008.

[6] E. Wigner. On the quantum correction for thermodynamic equilibrium. Phys. Rev., 40:749-759, Jun 1932.

[7] Foisal Siddiki, A Mamun, and M Amin. Modulational instability of quantum elctron-acoustic waves and associated envelope solitons in a degenerate quantum plasma. 112016.

[8] Bengt Eliasson and Padma Kant Shukla. Dispersion properties of electrostatic oscillations in quantum plasmas. Journal of Plasma Physics, 76(1):7-17, 2010.

[9] PK Shukla and Bengt Eliasson. Colloquium: Nonlinear collective interactions in quantum plasmas with degenerate electron fluids. Reviews of Modern Physics, 83(3):885, 2011.

[10] A. M. Abourabia and R. A. Shahein. Modulational instability and exact solutions of nonlinear cubic complex ginzburg-landau equation of thermodynamically open and dissipative warm ion acoustic waves system. The European Physical Journal Plus, 126(2):23, Feb 2011.
[11] Shatadru Chaudhuri and A RoyChowdhury. Wigner transform and stability of kinetic envelope soliton in an electron depleted dusty plasma with two temperature non-thermal ions. Physica Scripta, 94(3):035601, Jan 2019.

[12] Shatadru Chaudhuri and A RoyChowdhury. Nonlinear Landau damping in a relativistic electron-ion plasma-non-local nonlinear Schrödinger-equation and Krylov Bogoliubov Mitropolsky method. Physica Scripta, 93(7):075601, Jun 2018.

[13] A.; Wheatland M. S. Keane, A. J.; Mushtaq. Alfvén solitons in a fermionic quantum plasma. Physical Review E, 83, 62011.

[14] A. Roy Chowdhury and G. Pakira. On a kinetic approach to the modulational stability of envelope solitons in a relativistic plasma in an external electric field. Il Nuovo Cimento D, 14(5):527-539, May 1992.

[15] Nusrat Jehan, M. Salahuddin, and Arshad M. Mirza. Perpendicular propagating electromagnetic envelope solitons in electron-positron-ion plasma. Physics of Plasmas, 17(5):052308, 2010.

[16] N. Akhtar, W. F. El-Taibany, S. Mahmood, E. E. Behery, S. A. Khan, S. Ali, and S. Hussain. Transverse instability of ion acoustic solitons in a magnetized plasma including $q$-nonextensive electrons and positrons. Journal of Plasma Physics, 81(5):905810518, 2015.

[17] NN Bogoliubov and Yu Mitropolskii. A., 1961, asymptotic methods in the theory of nonlinear oscillations. Eng. Trans.](Delhi: Hindustan Publishing Corporation) $p, 51$.

[18] NN Bogoliubov and Yu A Mitropolsky. Asymptotic methods in the theory of nonlinear oscillations gordon and breach. New York, 1961.

[19] T. Brooke Benjamin and J. E. Feir. The disintegration of wave trains on deep water part 1 . theory. Journal of Fluid Mechanics, 27(3):417-430, 1967.

[20] V. I.Bespalov and V.I.Talanov. (3):417, 1966. 